\title{
Balanced judicious bipartitions of graphs
}

\author{
Baogang $\mathrm{Xu}{ }^{a, *}, \quad J u a n \operatorname{Yan}^{a, b}$ \\ ${ }^{a}$ School of Mathematics and Computer Science \\ Nanjing Normal University, 122 Ninghai Road, Nanjing, 210097, China \\ ${ }^{b}$ College of Mathematics and System Sciences \\ Xinjiang University, Urumqi, Xinjiang 830046, China \\ Xingxing $\mathrm{Yu}^{\dagger}$ \\ School of Mathematics \\ Georgia Institute of Technology \\ Atlanta, GA 30332-0160, USA
}

\begin{abstract}
A bipartition of the vertex set of a graph is called balanced if the sizes of the sets in the bipartition differ by at most one. Bollobás and Scott [3] conjectured that if $G$ is a graph with minimum degree at least 2 then $V(G)$ admits a balanced bipartition $V_{1}, V_{2}$ such that for each $i, G$ has at most $|E(G)| / 3$ edges with both ends in $V_{i}$. The minimum degree condition is necessary, and a result of Bollobás and Scott [5] shows that this conjecture holds for regular graphs $G$ (i.e., when $\Delta(G)=\delta(G)$ ). We prove this conjecture for graphs $G$ with $\Delta(G) \leq \frac{7}{5} \delta(G)$; hence it holds for graphs $G$ with $\delta(G) \geq \frac{5}{7}|V(G)|$.
\end{abstract}

Key words and phrases: Balanced partition, judicious partition, maximum degree, minimum degree

AMS 2000 Subject Classifications: 05C35, 05C75

*Partially supported by NSFC 10671095. Email: baogxu@njnu.edu.cn

${ }^{\dagger}$ Partially supported by NSA and by NSFC Project 16028102. Email: yu@math.gatech.edu 


\section{Introduction}

The Maximum Bipartite Subgraph Problem is a classical partition problem which optimizes one quantity: Given a graph $G$, find a partition of $V(G)$ into $V_{1}, V_{2}$ that minimizes $e\left(V_{1}\right)+e\left(V_{2}\right)$, where $e\left(V_{i}\right)(i \in\{1,2\})$ denotes the number of edges of $G$ with both ends in $V_{i}$. A simple calculation shows that every graph with $m$ edges has a bipartite subgraph with at least $m / 2$ edges. Edwards [6,7] improved this lower bound to $\frac{m}{2}+\frac{1}{4} \sqrt{2 m+\frac{1}{4}}-\frac{1}{8}$, which is essentially best possible as evidenced by the complete graphs $K_{2 n+1}$. In [4] (also see [3]), Bollobás and Scott extend Edwards' bound to $k$-partitions of graphs by proving that the vertex set of any graph with $m$ edges can be partitioned into $V_{1}, \ldots, V_{k}$ such that $e\left(V_{1}, \ldots, V_{k}\right) \geq \frac{k-1}{k} m+$ $\frac{k-1}{2 k} \sqrt{2 m+\frac{1}{4}}+O\left(k^{2}\right)$, where $e\left(V_{1}, \ldots, V_{k}\right)$ denotes the number of edges of $G$ that join vertices from different sets.

Judicious partition problems ask for a partition of the vertex set of a graph into subsets so that several quantities are optimized simultaneously. The Bottleneck Bipartition Problem, introduced by Entringer (see [10]), is such an example: Given a graph $G$, find a partition $V_{1}, V_{2}$ of $V(G)$ that minimizes $\max \left\{e\left(V_{1}\right), e\left(V_{2}\right)\right\}$. Székely and Shahrokhi [10] showed that this problem is NP-hard. Porter [8] proved that for any graph $G$ with $m$ edges there is a partition $V_{1}, V_{2}$ of $V(G)$ such that $\max \left\{e\left(V_{1}\right), e\left(V_{2}\right)\right\} \leq m / 4+O(\sqrt{m})$, establishing a conjecture of Erdös. (A matrix version of this Erdös conjecture was formulated by Entringer, and was solved by Porter and Székely [9].)

The Bottleneck Bipartition Problem was also studied by Bollobás and Scott [1,2]; they show in [2] that for any graph $G$ with $m$ edges there is a bipartition $V_{1}, V_{2}$ of $V(G)$ such that $e\left(V_{1}, V_{2}\right) \geq \frac{m}{2}+\frac{1}{4} \sqrt{2 m+\frac{1}{4}}-\frac{1}{8}$ and $\max \left\{e\left(V_{1}\right), e\left(V_{2}\right)\right\} \leq \frac{m}{4}+\frac{1}{8} \sqrt{2 m+\frac{1}{4}}-\frac{1}{16} . \mathrm{Xu}$ and $\mathrm{Yu}[11]$ extended this result to $k$-partitions (for $k \geq 3$ ), answering a question of Bollobás and Scott [3]: The vertex set of any graph with $m$ edges can be partitioned into $V_{1}, \ldots, V_{k}$ such that $e\left(V_{i}\right) \leq$ $\frac{m}{k^{2}}+\frac{k-1}{2 k^{2}}\left(\sqrt{2 m+\frac{1}{4}}-\frac{1}{2}\right)$ for $i \in\{1,2, \ldots, k\}$, and $e\left(V_{1}, \ldots, V_{k}\right) \geq \frac{k-1}{k} m+\frac{1}{2 k}\left(\sqrt{2 m+\frac{1}{4}}-\frac{1}{2}\right)$.

This paper concerns the Bottleneck Bipartiton Problem with an additional requirement on the bipartitions. A $k$-partition $V_{1}, \ldots, V_{k}$ of $V(G)$ is said to be balanced if $-1 \leq\left|V_{i}\right|-$ $\left|V_{j}\right| \leq 1$ for $1 \leq i, j \leq k$; the classical Min $k$-Section Problem asks for such a partition that minimizes $e\left(V_{1}, \ldots, V_{k}\right)$. Bollobás and Scott [3] asked an analogous question for judicious partitions: Given a graph $G$, find a balanced partition of $V(G)$ into $V_{1}, \ldots, V_{k}$ that minimizes $\max \left\{e\left(V_{1}\right), \ldots, e\left(V_{k}\right)\right\}$. In particular, they made the following conjecture, where $e(G)$ denotes the number of edges in the graph $G$.

Conjecture 1.1 (Bollobás and Scott [3]) Let $G$ be a graph with minimum degree at least 2. Then $V(G)$ admits a balanced partition $V_{1}, V_{2}$ such that $e\left(V_{i}\right) \leq e(G) / 3$ for $i \in\{1,2\}$.

The complete graph $K_{3}$ shows that the bound $e(G) / 3$ is sharp. The star $K_{1, n}$ shows that the requirement on minimum degree is necessary (otherwise, one cannot do better than $e(G) / 2$ in general). Bollobás and Scott [5] proved the following result, which implies Conjecture 1.1 for regular graphs.

Theorem 1.2 (Bollobás and Scott [5]) Let $d \geq 2$ be an integer, and let $G$ be a d-regular graph. Then $V(G)$ admits a balanced bipartition $V_{1}, V_{2}$ such that

(1) $e\left(V_{i}\right) \leq \frac{1}{4} \frac{d-1}{d} e(G)$ when $d$ is odd, 
(2) $e\left(V_{i}\right) \leq \frac{1}{4} \frac{d}{d+1} e(G)$ when $d$ is even and $|V(G)|$ is even, and

(3) $e\left(V_{i}\right) \leq \frac{1}{4} \frac{d}{d+1} e(G)+\frac{d}{4}$ when $d$ is even and $|V(G)|$ is odd.

Moreover, the extremal graphs for (1) are $s K_{d+1}$ for $s \geq 1$, those for (2) are $2 s K_{d+1}$ for $s \geq 1$, and those for (3) are $(2 s+1) K_{d+1}$ for $s \geq 0$.

For a graph $G$, we use $\Delta(G)$ and $\delta(G)$ to denote the maximum and minimum degree of $G$, respectively. So a graph $G$ is regular iff $\Delta(G)-\delta(G)=0$. The following result of Yan and $\mathrm{Xu}$ [12] generalizes Theorem 1.2 to graphs $G$ with $\Delta(G)-\delta(G)=1$.

Theorem 1.3 (Yan and $X u$ [12J) Let $d \geq 2$ be an integer, and let $G$ be a graph with $n_{1}$ vertices of degree $d$ and $n_{2}:=|V(G)|-n_{1}$ vertices of degree $d-1$. Then $V(G)$ admits a balanced bipartition $V_{1}, V_{2}$ such that

(1) $e\left(V_{i}\right) \leq e(G) / 4-n_{1} / 8$ when $d$ is odd and $|V(G)|$ is even,

(2) $e\left(V_{i}\right) \leq e(G) / 4-n_{1} / 8+(d-1) / 8$ when $d$ is odd and $|V(G)|$ is odd,

(3) $e\left(V_{i}\right) \leq e(G) / 4+n_{2} / 8$ when $d$ is even and $|V(G)|$ is even,

(4) $e\left(V_{i}\right) \leq e(G) / 4+n_{2} / 8+d / 8$ when $d$ is even and $|V(G)|$ is odd.

The main goal of this paper is to provide further evidence to Conjecture 1.1, by proving it for graphs $G$ for which $\Delta(G)-\delta(G)$ is not too large.

Theorem 1.4 Let $G$ be a graph, and assume that $\Delta(G) \leq \frac{7}{5} \delta(G)$. Then $G$ admits a balanced partition $V_{1}, V_{2}$ such that $e\left(V_{i}\right) \leq e(G) / 3$ for $i \in\{1,2\}$.

Since $\Delta(G) \leq|V(G)|-1, \delta(G) \geq 5|V(G)| / 7$ implies $\Delta(G) \leq \frac{7}{5} \delta(G)$. So we have the following immediate consequence of Theorem 1.4, which implies Conjecture 1.1 for graphs $G$ with $\delta(G) \geq 5|V(G)| / 7$.

Corollary 1.5 Let $G$ be a graph with $\delta(G) \geq 5|V(G)| / 7$. Then $V(G)$ admits a balanced partition $V_{1}, V_{2}$ such that $e\left(V_{i}\right) \leq e(G) / 3$ for $i \in\{1,2\}$.

Theorems 1.2, 1.3 and 1.4 suggest that the bound on $e\left(V_{i}\right)$ in Conjecture 1.1 decrease from $e(G) / 3$ to $e(G) / 4$ as $\Delta(G)$ decreases from $\frac{7}{5} \delta(G)$ to $\delta(G)$. Indeed, the next result shows that this may be the case: The bound on $\max \left\{e\left(V_{1}\right), e\left(V_{2}\right)\right\}$ decreases from $e(G) / 2$ to $e(G) / 4$ as $\Delta(G)$ decreases from $3 \delta(G)$ to $\delta(G)$. Note that $(r+4) /(3 r-4)$ takes on the values $3,7 / 5,1$ when $r=2,3,4$, respectively.

Theorem 1.6 Let $2 \leq r \leq 4$ be a real number, and let $G$ be a graph. Suppose $\Delta(G) \leq$ $\frac{r+4}{3 r-4} \delta(G)$ when $|V(G)|$ is even, and $\Delta(G) \leq \frac{r+4}{3 r-4} \delta(G)-\frac{4 r}{3 r-4}$ when $|V(G)|$ is odd. Then $V(G)$ admits a balanced partition $V_{1}, V_{2}$ such that $e\left(V_{i}\right) \leq e(G) / r$ for $i \in\{1,2\}$.

The rest of this paper is organized as follows. In Section 2, we prove several lemmas. In Section 3 we prove Theorems 1.4 and 1.6. Section 4 contains remarks and further questions. 


\section{Lemmas}

In this section, we prove three lemmas to be used in the proofs of Theorems 1.4 and 1.6. Let $G$ be a graph and let $V_{1}, V_{2}$ be a partition of $V(G)$. For $j \in\{1,2\}$ and $i \in\{\delta(G), \delta(G)+$ $1, \ldots, \Delta(G)\}$, we let $n_{j, i}$ denote the number of vertices in $V_{j}$ that have degree $i$ in $G$. When there is no possibility of confusion, we write $\delta$ and $\Delta$ instead of $\delta(G)$ and $\Delta(G)$.

Note that for $\delta \leq i \leq \Delta, 0 \leq \Delta-i \leq \Delta-\delta$. We have the following simple observations for $j \in\{1,2\}$ :

Observation (a). $\sum_{i=\delta}^{\Delta} n_{j, i}=\sum_{i=0}^{\Delta-\delta} n_{j, \Delta-i}=\left|V_{j}\right|$;

Observation (b). $\sum_{i=0}^{\Delta-\delta} i n_{j, \Delta-i}=\sum_{i=0}^{\Delta-\delta} \Delta n_{j, \Delta-i}-\sum_{i=0}^{\Delta-\delta}(\Delta-i) n_{j, \Delta-i} \leq(\Delta-\delta)\left|V_{j}\right|$.

The first two lemmas express and estimate $e\left(V_{i}\right)$ in terms of $n_{j, i}$.

Lemma 2.1 Let $G$ be a graph, and let $V_{1}, V_{2}$ be a bipartition of $V(G)$. Then,

(i) $e(G)=\frac{1}{2}\left(\Delta|V(G)|-\sum_{i=1}^{\Delta-\delta} i n_{1, \Delta-i}-\sum_{i=1}^{\Delta-\delta} i n_{2, \Delta-i}\right)$.

(ii) $e\left(V_{1}\right)-e\left(V_{2}\right)=\frac{1}{2} \sum_{i=1}^{\Delta-\delta} i\left(n_{2, \Delta-i}-n_{1, \Delta-i}\right)-\frac{\Delta}{2}\left(\left|V_{2}\right|-\left|V_{1}\right|\right)$.

Proof. By the Handshaking Lemma,

$$
\begin{aligned}
2 e(G) & =\sum_{i=\delta}^{\Delta} i\left(n_{1, i}+n_{2, i}\right) \\
& =\sum_{i=\delta}^{\Delta} \Delta\left(n_{1, i}+n_{2, i}\right)-\sum_{i=\delta}^{\Delta-1}(\Delta-i)\left(n_{1, i}+n_{2, i}\right) \\
& =\Delta\left(\left|V_{1}\right|+\left|V_{2}\right|\right)-\sum_{i=1}^{\Delta-\delta} i\left(n_{1, \Delta-i}+n_{2, \Delta-i}\right) \quad \text { (by Observation (a)) } \\
& =\Delta|V(G)|-\sum_{i=1}^{\Delta-\delta} i n_{1, \Delta-i}-\sum_{i=1}^{\Delta-\delta} i n_{2, \Delta-i},
\end{aligned}
$$

which proves $(i)$. Since

$$
2 e\left(V_{1}\right)+e\left(V_{1}, V_{2}\right)=\sum_{i=\delta}^{\Delta} i n_{1, i}
$$

and

$$
2 e\left(V_{2}\right)+e\left(V_{1}, V_{2}\right)=\sum_{i=\delta}^{\Delta} i n_{2, i}
$$




$$
\begin{aligned}
e\left(V_{1}\right)-e\left(V_{2}\right) & =\frac{1}{2} \sum_{i=\delta}^{\Delta} i\left(n_{1, i}-n_{2, i}\right) \\
& =\frac{1}{2} \sum_{i=0}^{\Delta-\delta}(\Delta-i)\left(n_{1, \Delta-i}-n_{2, \Delta-i}\right) \\
& =\frac{1}{2}\left(\sum_{i=0}^{\Delta-\delta} i\left(n_{2, \Delta-i}-n_{1, \Delta-i}\right)+\Delta \sum_{i=0}^{\Delta-\delta} n_{1, \Delta-i}-\Delta \sum_{i=0}^{\Delta-\delta} n_{2, \Delta-i}\right) .
\end{aligned}
$$

Therefore, (ii) follows from Observation (a).

Lemma 2.2 Let $G$ be a graph, and let $V_{1}, V_{2}$ be a balanced bipartition of $V(G)$ such that $e\left(V_{1}, V_{2}\right)$ is maximum among all balanced bipartitions of $V(G)$. For $v \in V(G)$ let $t_{v}:=$ $\left|N(v) \cap V_{1}\right|-\left|N(v) \cap V_{2}\right|$, and let $t:=\max \left\{t_{v}: v \in V_{1}\right\}$. Then

(i) $e\left(V_{1}\right) \leq \frac{\Delta+t}{4}\left|V_{1}\right|-\frac{1}{4} \sum_{i=1}^{\Delta-\delta} i n_{1, \Delta-i}$.

(ii) $e\left(V_{2}\right) \leq \frac{\Delta-t}{4}\left|V_{2}\right|-\frac{1}{4} \sum_{i=1}^{\Delta-\delta} i n_{2, \Delta-i}$

Proof. First, we estimate $e\left(V_{1}\right)$. Note that for $v \in V_{1}, t_{v}=\left|N(v) \cap V_{1}\right|-\left|N(v) \cap V_{2}\right| \leq t$ and $\left|N(v) \cap V_{1}\right|+\left|N(v) \cap V_{2}\right|=d(v)$. So $\left|N(v) \cap V_{1}\right| \leq \frac{d(v)+t}{2}$, and hence

$$
\begin{aligned}
2 e\left(V_{1}\right) & =\sum_{v \in V_{1}}\left|N(v) \cap V_{1}\right| \\
& \leq \frac{\Delta+t}{2} n_{1, \Delta}+\frac{(\Delta-1)+t}{2} n_{1, \Delta-1}+\ldots+\frac{\delta+t}{2} n_{1, \delta} \\
& =\frac{\Delta+t}{2}\left|V_{1}\right|-\frac{1}{2} \sum_{i=1}^{\Delta-\delta} i n_{1, \Delta-i} \quad \text { (by Observation (a)) }
\end{aligned}
$$

which implies $(i)$.

Next we estimate $e\left(V_{2}\right)$. Let $v_{1} \in V_{1}$ with $t_{v_{1}}=t$.

Suppose for the moment that there exists $v_{2} \in V_{2}$ such that $t_{v_{2}}=\left|N\left(v_{2}\right) \cap V_{1}\right|-\mid N\left(v_{2}\right) \cap$ $V_{2} \mid<t=t_{v_{1}}$. Define $V_{1}^{\prime}:=\left(V_{1} \backslash\left\{v_{1}\right\}\right) \cup\left\{v_{2}\right\}$ and $V_{2}^{\prime}:=\left(V_{2} \backslash\left\{v_{2}\right\}\right) \cup\left\{v_{1}\right\}$. Then $V_{1}^{\prime}, V_{2}^{\prime}$ is also a balanced bipartition of $V(G)$, and

$$
\begin{aligned}
e\left(V_{1}^{\prime}, V_{2}^{\prime}\right) & \geq e\left(V_{1}, V_{2}\right)+\left(\left|N\left(v_{1}\right) \cap V_{1}\right|-\left|N\left(v_{1}\right) \cap V_{2}\right|\right)-\left(\left|N\left(v_{2}\right) \cap V_{1}\right|-\left|N\left(v_{2}\right) \cap V_{2}\right|\right) \\
& =e\left(V_{1}, V_{2}\right)+t_{v_{1}}-t_{v_{2}} \\
& \geq e\left(V_{1}, V_{2}\right)+1
\end{aligned}
$$

which contradicts the maximality of $e\left(V_{1}, V_{2}\right)$. 
Therefore, for all $w \in V_{2}, t_{w}=\left|N(w) \cap V_{1}\right|-\left|N(w) \cap V_{2}\right| \geq t$. Since $\left|N(w) \cap V_{1}\right|+\mid N(w) \cap$ $V_{2} \mid=d(w)$, we have $\left|N(w) \cap V_{2}\right| \leq \frac{d(w)-t}{2}$. Therefore,

$$
\begin{aligned}
2 e\left(V_{2}\right) & \leq \frac{\Delta-t}{2} n_{2, \Delta}+\frac{\Delta-1-t}{2} n_{2, \Delta-1}+\cdots+\frac{\delta-t}{2} n_{2, \delta} \\
& =\frac{\Delta-t}{2}\left|V_{2}\right|-\frac{1}{2} \sum_{i=1}^{\Delta-\delta} i n_{2, \Delta-i} \quad(\text { by Observation (a)) }
\end{aligned}
$$

which implies $(i i)$.

The next lemma implies Theorems 1.4 and 1.6 for graphs of even order. The technique is similar to that used in [5], by considering a balanced partition $V_{1}, V_{2}$ that maximizes $e\left(V_{1}, V_{2}\right)$.

Lemma 2.3 Let $2 \leq r \leq 4$ be a real number, and let $G$ be a graph such that $|V(G)|$ is even and $\Delta(G) \leq \frac{r+4}{3 r-4} \delta(G)$. Then $V(G)$ admits a balanced bipartition $V_{1}, V_{2}$ such that $e\left(V_{i}\right) \leq e(G) / r$ for $i \in\{1,2\}$.

Proof. Let $V_{1}, V_{2}$ be a balanced bipartition of $V(G)$ such that $e\left(V_{1}, V_{2}\right)$ is maximum among all balanced bipartitions of $V(G)$. Then $\left|V_{1}\right|=\left|V_{2}\right|=|V(G)| / 2$. Without loss of generality, we may assume that $e\left(V_{1}\right) \geq e\left(V_{2}\right)$. If $e\left(V_{1}\right) \leq e(G) / r$ then the assertion of the lemma holds. So we may assume that $e\left(V_{1}\right)>e(G) / r$.

Let $t_{v}:=\left|N(v) \cap V_{1}\right|-\left|N(v) \cap V_{2}\right|$ (for all $v \in V(G)$ ) and define $t:=\max \left\{t_{v}: v \in V_{1}\right\}$. By Lemma 2.2(i) and the fact $\left|V_{1}\right|=|V(G)| / 2$,

$$
e\left(V_{1}\right) \leq\left(\frac{\Delta+t}{4}\right) \frac{|V(G)|}{2}-\frac{1}{4} \sum_{i=1}^{\Delta-\delta} i n_{1, \Delta-i},
$$

where $\Delta:=\Delta(G)$ and $\delta:=\delta(G)$. By Lemma 2.1(i) and the assumption $e\left(V_{1}\right)>e(G) / r$,

$$
\frac{1}{2 r}\left(\Delta|V(G)|-\sum_{i=1}^{\Delta-\delta} i n_{1, \Delta-i}-\sum_{i=1}^{\Delta-\delta} i n_{2, \Delta-i}\right)<\left(\frac{\Delta+t}{4}\right) \frac{|V(G)|}{2}-\frac{1}{4} \sum_{i=1}^{\Delta-\delta} i n_{1, \Delta-i} .
$$

Hence

$$
\begin{aligned}
& 4 \Delta|V(G)| \\
< & r(\Delta+t)|V(G)|-2(r-2) \sum_{i=1}^{\Delta-\delta} i n_{1, \Delta-i}+4 \sum_{i=1}^{\Delta-\delta} i n_{2, \Delta-i} \\
\leq & r(\Delta+t)|V(G)|+4 \sum_{i=1}^{\Delta-\delta} i n_{2, \Delta-i} \quad(\text { since } r \geq 2) \\
\leq & \left.r(\Delta+t)|V(G)|+2(\Delta-\delta)|V(G)| \quad \text { (by Observation (b) and the fact }\left|V_{2}\right|=\frac{|V(G)|}{2}\right) .
\end{aligned}
$$

Therefore,

$$
t>\frac{(2-r) \Delta+2 \delta}{r}
$$


By Lemma 2.1(ii) and that fact $\left|V_{1}\right|=\left|V_{2}\right|$,

$$
e\left(V_{1}\right)-e\left(V_{2}\right)=\frac{1}{2} \sum_{i=1}^{\Delta-\delta} i\left(n_{2, \Delta-i}-n_{1, \Delta-i}\right)
$$

So it follows from Lemma $2.2(i i)$ and that fact $\left|V_{2}\right|=|V(G)| / 2$ that

$$
e\left(V_{1}\right) \leq\left(\frac{\Delta-t}{4}\right) \frac{|V(G)|}{2}-\frac{1}{4} \sum_{i=1}^{\Delta-\delta} i n_{2, \Delta-i}+\frac{1}{2} \sum_{i=1}^{\Delta-\delta} i\left(n_{2, \Delta-i}-n_{1, \Delta-i}\right) .
$$

Then, by Lemma $2.1(i)$ and the assumption $e\left(V_{1}\right)>e(G) / r$, we have

$$
\begin{aligned}
& \frac{1}{2 r}\left(\Delta|V(G)|-\sum_{i=1}^{\Delta-\delta} i n_{1, \Delta-i}-\sum_{i=1}^{\Delta-\delta} i n_{2, \Delta-i}\right) \\
< & \frac{\Delta-t}{4} \frac{|V(G)|}{2}-\frac{1}{4} \sum_{i=1}^{\Delta-\delta} i n_{2, \Delta-i}+\frac{1}{2} \sum_{i=1}^{\Delta-\delta} i\left(n_{2, \Delta-i}-n_{1, \Delta-i}\right) .
\end{aligned}
$$

Thus

$$
\begin{aligned}
& 4 \Delta|V(G)| \\
< & r(\Delta-t)|V(G)|+2(r+2) \sum_{i=1}^{\Delta-\delta} i n_{2, \Delta-i}-4(r-1) \sum_{i=1}^{\Delta-\delta} i n_{1, \Delta-i} \\
\leq & r(\Delta-t)|V(G)|+2(r+2) \sum_{i=1}^{\Delta-\delta} i n_{2, \Delta-i} \quad(\text { since } r \geq 2) \\
\leq & r(\Delta-t)|V(G)|+(r+2)(\Delta-\delta)|V(G)| \quad\left(\text { by Observation (b) and since }\left|V_{2}\right|=\frac{|V(G)|}{2}\right) \\
< & \left(r\left(\Delta-\frac{(2-r) \Delta+2 \delta}{r}\right)+(r+2)(\Delta-\delta)\right)|V(G)| \quad(\text { by }(1)) \\
= & (3 r \Delta-(r+4) \delta)|V(G)| .
\end{aligned}
$$

Therefore,

$$
\Delta>\frac{r+4}{3 r-4} \delta
$$

a contradiction to the assumption that $\Delta \leq \frac{r+4}{3 r-4} \delta$.

\section{Proof of Theorems 1.4 and $\mathbf{1 . 6}$}

Proof of Theorem 1.4. By Lemma 2.3 (with $r=3$ ), we see that the assertion of Theorem 1.4 holds when $|V(G)|$ is even. So we may assume that $|V(G)|$ is odd.

Let $V_{1}, V_{2}$ be a balanced bipartition of $V(G)$ such that $e\left(V_{1}, V_{2}\right)$ is maximum among all balanced bipartitions of $V(G)$. Without loss of generality, we may assume that $e\left(V_{1}\right) \geq e\left(V_{2}\right)$. If $e\left(V_{1}\right) \leq e(G) / 3$, the assertion of Theorem 1.4 holds. So we may assume $e\left(V_{1}\right)>e(G) / 3$. This, in particular, implies that $e\left(V_{1}, V_{2}\right)<2 e(G) / 3$. 
We claim that there exists $v_{1} \in V_{1}$ such that $\left|N\left(v_{1}\right) \cap V_{1}\right|>\left|N\left(v_{1}\right) \cap V_{2}\right|$. For, otherwise, $\left|N(v) \cap V_{1}\right| \leq\left|N(v) \cap V_{2}\right|$ for all $v \in V_{1}$. Hence

$$
\begin{aligned}
2 e\left(V_{1}\right) & =\sum_{v \in V_{1}}\left|N(v) \cap V_{1}\right| \\
& \leq \sum_{v \in V_{1}}\left|N(v) \cap V_{2}\right| \\
& =e\left(V_{1}, V_{2}\right) \\
& <2 e(G) / 3 .
\end{aligned}
$$

This is a contradiction to the assumption that $e\left(V_{1}\right)>e(G) / 3$.

Since $V_{1}, V_{2}$ is a balanced bipartition of $V(G)$, and since $n:=|V(G)|$ is odd, either $\left|V_{1}\right|=\frac{n-1}{2}$ or $\left|V_{1}\right|=\frac{n+1}{2}$. Indeed,

$$
\left|V_{1}\right|=\frac{n-1}{2} \text { and }\left|V_{2}\right|=\frac{n+1}{2} .
$$

For, otherwise, $V_{1}^{\prime}:=V_{1} \backslash\left\{v_{1}\right\}, V_{2}^{\prime}:=V_{2} \cup\left\{v_{1}\right\}$ is also a balanced bipartition of $V(G)$, and $e\left(V_{1}^{\prime}, V_{2}^{\prime}\right)=e\left(V_{1}, V_{2}\right)+\left|N\left(v_{1}\right) \cap V_{1}\right|-\left|N\left(v_{1}\right) \cap V_{2}\right| \geq e\left(V_{1}, V_{2}\right)+1$. But this contradicts the maximality of $e\left(V_{1}, V_{2}\right)$.

Let $t_{v}:=\left|N(v) \cap V_{1}\right|-\left|N(v) \cap V_{2}\right|$ (for all $v \in V(G)$ ) and define $t:=\max \left\{t_{v}: v \in V_{1}\right\}$. By Lemma 2.1(i) and Lemma 2.2(i), and by the assumption that $e\left(V_{1}\right)>e(G) / 3$, we have

$$
\frac{1}{3}\left(\Delta n-\sum_{i=1}^{\Delta-\delta} i n_{1, \Delta-i}-\sum_{i=1}^{\Delta-\delta} i n_{2, \Delta-i}\right)<\left(\frac{\Delta+t}{2}\right)\left(\frac{n-1}{2}\right)-\frac{1}{2} \sum_{i=1}^{\Delta-\delta} i n_{1, \Delta-i} .
$$

Hence

$$
\begin{aligned}
\Delta n & <\frac{3}{4}(\Delta+t)(n-1)+\sum_{i=1}^{\Delta-\delta} i n_{2, \Delta-i}-\frac{1}{2} \sum_{i=1}^{\Delta-\delta} i n_{1, \Delta-i} \\
& \leq \frac{3}{4}(\Delta+t)(n-1)+\sum_{i=1}^{\Delta-\delta} i n_{2, \Delta-i} \\
& \leq \frac{3}{4}(\Delta+t)(n-1)+(\Delta-\delta) \frac{n+1}{2} \quad \text { by Observation (b) and (2)) } \\
& =\frac{3(n-1) t}{4}+\frac{(5 n-1) \Delta-2(n+1) \delta}{4} .
\end{aligned}
$$

Therefore

$$
t>\frac{2(n+1) \delta-(n-1) \Delta}{3(n-1)}>\frac{2(n+1) \delta-(n-1) \Delta}{3 n} .
$$

By (2) and Lemma 2.1(ii),

$$
e\left(V_{1}\right)-e\left(V_{2}\right)=\frac{1}{2} \sum_{i=1}^{\Delta-\delta} i\left(n_{2, \Delta-i}-n_{1, \Delta-i}\right)-\frac{\Delta}{2} .
$$


So by Lemma 2.2(ii),

$$
\begin{aligned}
e\left(V_{1}\right) & \leq\left(\frac{\Delta-t}{4}\right)\left(\frac{n+1}{2}\right)-\frac{1}{4} \sum_{i=1}^{\Delta-\delta} i n_{2, \Delta-i}+\frac{1}{2} \sum_{i=1}^{\Delta-\delta} i\left(n_{2, \Delta-i}-n_{1, \Delta-i}\right)-\frac{\Delta}{2} \\
& \leq\left(\frac{\Delta-t}{4}\right) \frac{n}{2}-\frac{1}{4} \sum_{i=1}^{\Delta-\delta} i n_{2, \Delta-i}+\frac{1}{2} \sum_{i=1}^{\Delta-\delta} i\left(n_{2, \Delta-i}-n_{1, \Delta-i}\right)-\frac{3 \Delta+t}{8} .
\end{aligned}
$$

Therefore, it follows from Lemma $2.1(i)$ and the assumption $e\left(V_{1}\right)>e(G) / 3$ that

$$
\frac{1}{3}\left(\Delta n-\sum_{i=1}^{\Delta-\delta} i n_{1, \Delta-i}-\sum_{i=1}^{\Delta-\delta} i n_{2, \Delta-i}\right)<\frac{(\Delta-t) n}{4}+\frac{1}{2} \sum_{i=1}^{\Delta-\delta} i n_{2, \Delta-i}-\sum_{i=1}^{\Delta-\delta} i n_{1, \Delta-i}-\frac{3 \Delta+t}{4} .
$$

By rearranging and combining terms, we have

$$
\begin{aligned}
\Delta n & <\frac{3 n(\Delta-t)}{4}+\frac{5}{2} \sum_{i=1}^{\Delta-\delta} i n_{2, \Delta-i}-2 \sum_{i=1}^{\Delta-\delta} i n_{1, \Delta-i}-\frac{9 \Delta+3 t}{4} \\
& \leq \frac{3 n(\Delta-t)}{4}+\frac{5}{2} \sum_{i=1}^{\Delta-\delta} i n_{2, \Delta-i}-\frac{9 \Delta+3 t}{4} \\
& \leq \frac{(3 n-9) \Delta}{4}+\frac{5(n+1)(\Delta-\delta)}{4}-\frac{3(n+1) t}{4} \quad(\text { by }(2) \text { and Observation (b)) } \\
& <\frac{(3 n-9) \Delta}{4}+\frac{5(n+1)(\Delta-\delta)}{4}-\frac{3(n+1)}{4}\left(\frac{2(n+1) \delta-(n-1) \Delta}{3 n}\right) \quad(\text { by }(3)) \\
& =\frac{(3 n-9) \Delta}{4}+\frac{5(n+1)(\Delta-\delta)}{4}-\frac{2(n+1) \delta-(n-1) \Delta}{4}-\frac{2(n+1) \delta-(n-1) \Delta}{4 n} \\
& =\frac{4 n \Delta-10 \Delta}{4}+\frac{5 \Delta n+5 \Delta-7 \delta(n+1)}{4}-\frac{2(n+1) \delta-(n-1) \Delta}{4 n} \\
& =\frac{9 n \Delta-7 \delta(n+1)}{4}-\frac{2(n+1) \delta+4 n \Delta+\Delta}{4 n} \\
& <\frac{9 n \Delta-7 \delta(n+1)}{4} .
\end{aligned}
$$

Thus, $5 n \Delta>7(n+1) \delta>7 n \delta$. This implies $\Delta>7 \delta / 5$, a contradiction.

Proof of Theorem 1.6. By Lemma 2.3, the assertion of Theorem 1.6 holds when $|V(G)|$ is even. So we may assume that $n:=|V(G)|$ is odd.

Let $V_{1}, V_{2}$ be a balanced bipartition of $V(G)$ such that $e\left(V_{1}, V_{2}\right)$ is maximum among all balanced bipartitions of $V(G)$. Assume, without loss of generality, that $e\left(V_{1}\right) \geq e\left(V_{2}\right)$. If $e\left(V_{1}\right) \leq e(G) / r$ then the assertion of Theorem 1.6 holds. So we may assume that $e\left(V_{1}\right)>$ $e(G) / r$.

Let $t_{v}:=\left|N(v) \cap V_{1}\right|-\left|N(v) \cap V_{2}\right|$ (for all $v \in V(G)$ ), and define $t:=\max \left\{t_{v}: v \in V_{1}\right\}$. Since $\left|V_{1}\right|=\frac{n-1}{2}$ or $\left|V_{1}\right|=\frac{n+1}{2}$, we consider two cases.

Case 1. $\left|V_{1}\right|=\frac{n+1}{2}$ and $\left|V_{2}\right|=\frac{n-1}{2}$.

We claim that $t \leq 0$. For, if $t>0$, then there is $v \in V_{1}$ such that $t_{v}>0$. Now $V_{1}^{\prime}:=V_{1} \backslash\{v\}, V_{2}^{\prime}:=V_{2} \cup\{v\}$ is also a balanced bipartition of $V(G)$, and a simple calculation shows that $e\left(V_{1}^{\prime}, V_{2}^{\prime}\right)>e\left(V_{1}, V_{2}\right)$, contradicting the maximality of $e\left(V_{1}, V_{2}\right)$. 
By Lemma 2.2(i), we have

$$
e\left(V_{1}\right) \leq\left(\frac{\Delta+t}{4}\right)\left(\frac{n+1}{2}\right)-\frac{1}{4} \sum_{i=1}^{\Delta-\delta} i n_{1, \Delta-i} .
$$

Thus, by Lemma 2.1(i) and the assumption $e\left(V_{1}\right)>e(G) / r$,

$$
\frac{1}{2 r}\left(\Delta n-\sum_{i=1}^{\Delta-\delta} i n_{1, \Delta-i}-\sum_{i=1}^{\Delta-\delta} i n_{2, \Delta-i}\right)<\left(\frac{\Delta+t}{4}\right)\left(\frac{n+1}{2}\right)-\frac{1}{4} \sum_{i=1}^{\Delta-\delta} i n_{1, \Delta-i} .
$$

Hence

$$
\begin{aligned}
4 \Delta n & <r(\Delta+t)(n+1)-2(r-2) \sum_{i=1}^{\Delta-\delta} i n_{1, \Delta-i}+4 \sum_{i=1}^{\Delta-\delta} i n_{2, \Delta-i} \\
& \leq r(\Delta+t)(n+1)+4 \sum_{i=1}^{\Delta-\delta} i n_{2, \Delta-i} \quad(\text { since } r \geq 2) \\
& \leq r(\Delta+t)(n+1)+2(\Delta-\delta)(n-1) \quad \text { (by Observation (b)). }
\end{aligned}
$$

By Lemma 2.2(ii), we have

$$
e\left(V_{2}\right) \leq\left(\frac{\Delta-t}{4}\right)\left(\frac{n-1}{2}\right)-\frac{1}{4} \sum_{i=1}^{\Delta-\delta} i n_{2, \Delta-i} .
$$

By Lemma 2.1(ii),

$$
e\left(V_{1}\right)-e\left(V_{2}\right)=\frac{1}{2} \sum_{i=1}^{\Delta-\delta} i\left(n_{2, \Delta-i}-n_{1, \Delta-i}\right)+\frac{\Delta}{2} .
$$

These two expressions imply

$$
e\left(V_{1}\right) \leq\left(\frac{\Delta-t}{4}\right)\left(\frac{n-1}{2}\right)+\frac{1}{4} \sum_{i=1}^{\Delta-\delta} i n_{2, \Delta-i}-\frac{1}{2} \sum_{i=1}^{\Delta-\delta} i n_{1, \Delta-i}+\frac{\Delta}{2} .
$$

Thus, by Lemma 2.1 $(i)$ and the assumption $e\left(V_{1}\right)>e(G) / r$,

$$
\frac{1}{2 r}\left(\Delta n-\sum_{i=1}^{\Delta-\delta} i n_{1, \Delta-i}-\sum_{i=1}^{\Delta-\delta} i n_{2, \Delta-i}\right)<\left(\frac{\Delta-t}{4}\right)\left(\frac{n-1}{2}\right)+\frac{1}{4} \sum_{i=1}^{\Delta-\delta} i n_{2, \Delta-i}-\frac{1}{2} \sum_{i=1}^{\Delta-\delta} i n_{1, \Delta-i}+\frac{\Delta}{2} .
$$

Hence,

$$
\begin{aligned}
4 \Delta n & <r(\Delta-t)(n-1)+(2 r+4) \sum_{i=1}^{\Delta-\delta} i n_{2, \Delta-i}-(4 r-4) \sum_{i=1}^{\Delta-\delta} i n_{1, \Delta-i}+4 r \Delta \\
& \left.\leq r(\Delta-t)(n-1)+(2 r+4) \sum_{i=1}^{\Delta-\delta} i n_{2, \Delta-i}+4 r \Delta \quad \text { (since } r \geq 2\right) \\
& \leq r(\Delta-t)(n-1)+(r+2)(\Delta-\delta)(n-1)+4 r \Delta \quad \text { (by Observation (b)). }
\end{aligned}
$$


Since $\Delta \leq n-1,4(n+1) r \Delta \leq 4\left(n^{2}-1\right) r$. Multiplying (4) by $n-1$ and (5) by $n+1$, and combining the resulting inequalities, we have

$$
\begin{aligned}
8 \Delta n^{2} & <2 r \Delta\left(n^{2}-1\right)+2(\Delta-\delta)(n-1)^{2}+(r+2)(\Delta-\delta)\left(n^{2}-1\right)+4(n+1) r \Delta \\
& \leq 3 r \Delta n^{2}+4 n^{2} \Delta-(r+4) n^{2} \delta-4 n(\Delta-\delta)-(3 r \Delta-r \delta)+4\left(n^{2}-1\right) r \\
& =3 r n^{2} \Delta+4 n^{2} \Delta-(r+4) n^{2} \delta+4 r n^{2}-4 n(\Delta-\delta)-(3 r \Delta-r \delta+4 r) \\
& \leq 3 r n^{2} \Delta+4 n^{2} \Delta-(r+4) n^{2} \delta+4 r n^{2} .
\end{aligned}
$$

Therefore, $\Delta>\frac{r+4}{3 r-4} \delta-\frac{4 r}{3 r-4}$, a contradiction.

Case 2. $\left|V_{1}\right|=\frac{n-1}{2}$ and $\left|V_{2}\right|=\frac{n+1}{2}$.

By Lemma 2.1(ii),

$$
e\left(V_{1}\right)-e\left(V_{2}\right)=\frac{1}{2} \sum_{i=1}^{\Delta-\delta} i\left(n_{2, \Delta-i}-n_{1, \Delta-i}\right)-\frac{\Delta}{2} .
$$

By Lemma 2.2(ii),

$$
e\left(V_{2}\right) \leq\left(\frac{\Delta-t}{4}\right)\left(\frac{n+1}{2}\right)-\frac{1}{4} \sum_{i=1}^{\Delta-\delta} i n_{2, \Delta-i} .
$$

These two expressions imply

$$
e\left(V_{1}\right) \leq\left(\frac{\Delta-t}{4}\right)\left(\frac{n+1}{2}\right)+\frac{1}{4} \sum_{i=1}^{\Delta-\delta} i n_{2, \Delta-i}-\frac{1}{2} \sum_{i=1}^{\Delta-\delta} i n_{1, \Delta-i}-\frac{\Delta}{2} .
$$

Hence, by Lemma 2.1(i) and the assumption $e\left(V_{1}\right)>e(G) / r$, we have

$$
\frac{1}{2 r}\left(\Delta n-\sum_{i=1}^{\Delta-\delta} i n_{1, \Delta-i}-\sum_{i=1}^{\Delta-\delta} i n_{2, \Delta-i}\right)<\left(\frac{\Delta-t}{4}\right)\left(\frac{n+1}{2}\right)+\frac{1}{4} \sum_{i=1}^{\Delta-\delta} i n_{2, \Delta-i}-\frac{1}{2} \sum_{i=1}^{\Delta-\delta} i n_{1, \Delta-i}-\frac{\Delta}{2} .
$$

So

$$
\begin{aligned}
4 n \Delta & <r(\Delta-t)(n+1)+2(r+2) \sum_{i=1}^{\Delta-\delta} i n_{2, \Delta-i}-(4 r-4) \sum_{i=1}^{\Delta-\delta} i n_{1, \Delta-i}-4 r \Delta \\
& \leq r(\Delta-t)(n+1)+2(r+2) \sum_{i=1}^{\Delta-\delta} i n_{2, \Delta-i}-4 r \Delta \quad(\text { since } r \geq 2) \\
& \leq r(\Delta-t)(n+1)+(r+2)(\Delta-\delta)(n+1)-4 r \Delta \quad \text { (by Observation (b)). }
\end{aligned}
$$

By Lemma 2.2(i),

$$
e\left(V_{1}\right) \leq\left(\frac{\Delta+t}{4}\right)\left(\frac{n-1}{2}\right)-\frac{1}{4} \sum_{i=1}^{\Delta-\delta} i n_{1, \Delta-i}
$$


Therefore, by the assumption $e\left(V_{1}\right)>e(G) / r$ and by Lemma $2.1(i)$, we have

$$
\frac{1}{2 r}\left(\Delta n-\sum_{i=1}^{\Delta-\delta} i n_{1, \Delta-i}-\sum_{i=1}^{\Delta-\delta} i n_{2, \Delta-i}\right)<\left(\frac{\Delta+t}{4}\right)\left(\frac{n-1}{2}\right)-\frac{1}{4} \sum_{i=1}^{\Delta-\delta} i n_{1, \Delta-i} .
$$

So

$$
\begin{aligned}
4 n \Delta & <r(\Delta+t)(n-1)-2(r-2) \sum_{i=1}^{\Delta-\delta} i n_{1, \Delta-i}+4 \sum_{i=1}^{\Delta-\delta} i n_{2, \Delta-i} \\
& \leq r(\Delta+t)(n-1)+4 \sum_{i=1}^{\Delta-\delta} i n_{2, \Delta-i} \quad(\text { since } r \geq 2) \\
& \leq r(\Delta+t)(n-1)+2(\Delta-\delta)(n+1) \quad(\text { by Observation (b)). }
\end{aligned}
$$

Multiplying $(7)$ by $(n+1)$ and $(6)$ by $(n-1)$, and combining the resulting inequalities, we get

$$
\begin{aligned}
8 n^{2} \Delta & <2 r\left(n^{2}-1\right) \Delta+2(\Delta-\delta)(n+1)^{2}+(r+2)(\Delta-\delta)\left(n^{2}-1\right)-4(n-1) r \Delta \\
& =\left(3 r n^{2}+4 n^{2}+4 n+r-4 n r\right) \Delta-\left((r+4) n^{2}+4 n-r\right) \delta
\end{aligned}
$$

Hence

$$
\left((r+4) n^{2}+4 n-r\right) \delta<(3 r-4) n^{2} \Delta+(4 n+r-4 r n) \Delta,
$$

and so, $(r+4) n^{2} \delta<(3 r-4) n^{2} \Delta$. This implies $\Delta>\frac{r+4}{3 r-4} \delta$, a contradiction.

\section{Further discussions}

The proofs of Lemma 2.3 and Theorems 1.4 and 1.6 actually show that for any graph $G$ with $\Delta(G) \leq \frac{7}{5} \delta(G)$, any balanced bipartition $V_{1}, V_{2}$ of $V(G)$ with $e\left(V_{1}, V_{2}\right)$ maximum (among all balanced bipartitions) must satisfy $e\left(V_{i}\right) \leq e(G) / 3$. (The maximality of the partition makes it possible to derive the bound on $e\left(V_{i}\right)$, by allowing us to exchange some vertex of $V_{1}$ with a vertex of $V_{2}$.) Unfortunately, this is not always the case. For the graph $G$ in Figure 1, the bipartition $V_{1}:=\left\{x_{1}, \ldots, x_{7}\right\}, V_{2}:=\left\{y_{1}, \ldots, y_{7}\right\}$ of $V(G)$ is the unique balanced bipartition of $V(G)$ for which $e\left(V_{1}, V_{2}\right)$ is maximum. However, $e\left(V_{1}\right)=15>44 / 3=e(G) / 3$. Since it is not obvious why the partition $V_{1}, V_{2}$ is the unique maximum balanced bipartition of $V(G)$, we give a proof of this fact; which in a way indicates that when dealing with balanced bipartitions for general graphs, it is necessary to exchange subsets (of $V_{i}$ ) of size more than one.

Note that $G$ has a "reflection" symmetry in the line through the edge $x_{4} y_{4}$. Also note that $e\left(V_{2}\right)=0, e\left(V_{1}\right)=15$, and $e\left(V_{1}, V_{2}\right)=29$.

Let $V_{1}^{\prime}, V_{2}^{\prime}$ be an arbitrary balanced bipartition of $V(G)$ different from $V_{1}, V_{2}$. Then there exist $S_{i} \subseteq V_{i}, i=1,2$, with $0 \neq\left|S_{1}\right|=\left|S_{2}\right| \leq 3$ such that $V_{i}^{\prime}=\left(V_{i} \backslash S_{i}\right) \cup S_{3-i}$. We now proceed to show that $e\left(V_{1}^{\prime}, V_{2}^{\prime}\right)<e\left(V_{1}, V_{2}\right)$. Observe that

$$
\begin{aligned}
& e\left(V_{1}^{\prime}, V_{2}^{\prime}\right) \\
= & e\left(\left(V_{1} \backslash S_{1}\right) \cup S_{2},\left(V_{2} \backslash S_{2}\right) \cup S_{1}\right) \\
= & e\left(V_{1}, V_{2}\right)-e\left(S_{1}, V_{2} \backslash S_{2}\right)-e\left(S_{2}, V_{1} \backslash S_{1}\right)+e\left(S_{1}, V_{1} \backslash S_{1}\right) .
\end{aligned}
$$




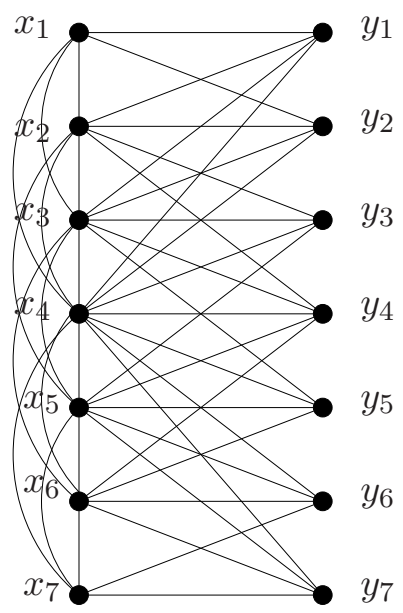

Figure 1: A graph with a unique maximum balanced bipartition.

So it suffices to show that

$$
e\left(S_{1}, V_{1} \backslash S_{1}\right)-e\left(S_{1}, V_{2} \backslash S_{2}\right)<e\left(S_{2}, V_{1} \backslash S_{1}\right) .
$$

Let $t_{j}:=\left|N\left(x_{j}\right) \cap V_{1}\right|-\left|N\left(x_{j}\right) \cap V_{2}\right|$ for $1 \leq j \leq 7$, and let $t\left(S_{1}\right):=\sum_{x_{j} \in S_{1}} t_{j}$. Then

$$
\begin{aligned}
& e\left(S_{1}, V_{1} \backslash S_{1}\right)-e\left(S_{1}, V_{2} \backslash S_{2}\right) \\
= & \left(\sum_{x_{j} \in S_{1}}\left|N\left(x_{j}\right) \cap V_{1}\right|\right)-2 e\left(S_{1}\right)-\left(\left(\sum_{x_{j} \in S_{1}}\left|N\left(x_{j}\right) \cap V_{2}\right|\right)-e\left(S_{1}, S_{2}\right)\right) \\
= & \left(\sum_{x_{j} \in S_{1}} t_{j}\right)-2 e\left(S_{1}\right)+e\left(S_{1}, S_{2}\right) \\
= & t\left(S_{1}\right)-2 e\left(S_{1}\right)+e\left(S_{1}, S_{2}\right) .
\end{aligned}
$$

Thus, it suffices to show that

$$
t\left(S_{1}\right)-2 e\left(S_{1}\right)+e\left(S_{1}, S_{2}\right)<e\left(S_{2}, V_{1} \backslash S_{1}\right) .
$$

We now list a few useful observations about the graph $G$ :

(1) $t_{j}=0$ for $j \in\{2,3,5,6\}, t_{1}=t_{7}=1$, and $t_{4}=-1$;

(2) $-1 \leq t\left(S_{1}\right) \leq 2$

(3) $t\left(S_{1}\right)=2$ iff $\left\{x_{1}, x_{7}\right\} \subseteq S_{1}$ and $x_{4} \notin S_{1}$;

(4) $t\left(S_{1}\right)=-1$ iff $x_{4} \in S_{1}$ and $\left\{x_{1}, x_{7}\right\} \cap S_{1}=\emptyset$;

(5) $e\left(S_{2}, V_{1} \backslash S_{1}\right) \geq 4\left|S_{2}\right|-e\left(S_{2}, S_{1}\right)$. 
If $\left|S_{1}\right|=\left|S_{2}\right|=1$, then $e\left(S_{1}, S_{2}\right) \leq 1, e\left(S_{1}\right)=0$, and $t\left(S_{1}\right) \leq 1$ (by (1)). Hence $t\left(S_{1}\right)-2 e\left(S_{1}\right)+e\left(S_{1}, S_{2}\right) \leq 1-0+1<4-1 \leq e\left(S_{2}, V_{1} \backslash S_{1}\right)$ (by (5)). So we may assume $\left|S_{1}\right|=\left|S_{2}\right| \in\{2,3\}$.

Case 1. $\left|S_{1}\right|=\left|S_{2}\right|=2$.

Then $e\left(S_{1}, S_{2}\right) \leq 4$ and $e\left(S_{1}\right) \leq 1$.

Suppose $e\left(S_{1}\right)=1$. Then $S_{1} \nsupseteq\left\{x_{1}, x_{7}\right\}$ (since $x_{1} x_{7} \notin E(G)$ ). It follows from (2) and (3) that $t\left(S_{1}\right) \leq 1$. Hence $t\left(S_{1}\right)-2 e\left(S_{1}\right)+e\left(S_{1}, S_{2}\right) \leq 1-2+4<e\left(S_{2}, V_{1} \backslash S_{1}\right)$ (by (5)).

Now assume $e\left(S_{1}\right)=0$. Then $x_{4} \notin S_{1}$ since $x_{4}$ is adjacent to all vertices (including those in $S_{1} \backslash\left\{x_{1}\right\}$; thus $t\left(S_{1}\right) \neq-1$ (by $\left.(4)\right)$.

If $t\left(S_{1}\right)=2$, then $S_{1}=\left\{x_{1}, x_{7}\right\}$ (by (3)). Since $x_{1}$ and $x_{7}$ have no common neighbor in $S_{2}, e\left(S_{1}, S_{2}\right) \leq 2$. Therefore $t\left(S_{1}\right)-2 e\left(S_{1}\right)+e\left(S_{1}, S_{2}\right) \leq 2-0+2<e\left(S_{2}, V_{1} \backslash S_{1}\right)$ (by (5)).

Assume $t\left(S_{1}\right)=1$. By (1), $x_{4} \notin S_{1}, S_{1} \cap\left\{x_{1}, x_{7}\right\} \neq \emptyset$ and $\left\{x_{2}, x_{3}, x_{5}, x_{6}\right\} \cap S_{1} \neq \emptyset$. By symmetry, we may assume that $x_{1} \in S_{1}$. Since $e\left(S_{1}\right)=0, S_{1}=\left\{x_{1}, x_{5}\right\}$ or $S_{1}=\left\{x_{1}, x_{6}\right\}$. Hence $e\left(S_{1}, S_{2}\right) \leq 2$, and so $t\left(S_{1}\right)-2 e\left(S_{1}\right)+e\left(S_{1}, S_{2}\right) \leq 1-0+2<e\left(S_{2}, V_{1} \backslash S_{1}\right)$ (by (5)).

So we may assume $t\left(S_{1}\right)=0$. Since $e\left(S_{1}\right)=0$ and $x_{4} \notin S_{1}$ and by (1), we have $S_{1}=$ $\left\{x_{2}, x_{6}\right\}$. So $e\left(S_{1}, S_{2}\right) \leq 3$ (since $\left|N\left(x_{2}\right) \cap N\left(x_{6}\right) \cap V_{2}\right|=1$ ), and hence $t\left(S_{1}\right)-2 e\left(S_{1}\right)+$ $e\left(S_{1}, S_{2}\right) \leq 0-0+3<e\left(S_{2}, V_{1} \backslash S_{1}\right)$ (by $\left.(5)\right)$.

Case 2. $\left|S_{1}\right|=\left|S_{2}\right|=3$.

Then $e\left(S_{1}, S_{2}\right) \leq 9$ and $e\left(S_{1}\right) \leq 3$. Also note that $e\left(S_{1}\right) \geq 1$.

First assume $e\left(S_{1}\right)=3$. Then, $\left\{x_{1}, x_{7}\right\} \nsubseteq S_{1}$, and hence $t\left(S_{1}\right) \leq 1$ by (2) and (3). If $t\left(S_{1}\right)=-1$, then $t\left(S_{1}\right)-2 e\left(S_{1}\right)+e\left(S_{1}, S_{2}\right) \leq-1-6+9<e\left(S_{2}, V_{1} \backslash S_{1}\right)$ (by (5)). So we assume $t\left(S_{1}\right) \geq 0$. It suffices to show $e\left(S_{1}, S_{2}\right) \leq 8$, since in that case $t\left(S_{1}\right)-2 e\left(S_{1}\right)+e\left(S_{1}, S_{2}\right) \leq$ $1-6+8<e\left(S_{2}, V_{1} \backslash S_{1}\right)$ (by (5)). This is clear if $\left\{x_{1}, x_{7}\right\} \cap S_{1} \neq \emptyset$, since $x_{1}$ and $x_{7}$ each have just two neighbors in $V_{2}$. So we may assume $\left\{x_{1}, x_{7}\right\} \cap S_{1}=\emptyset$. Then $t\left(S_{1}\right)=0$, and $S_{1} \subseteq\left\{x_{2}, x_{3}, x_{5}, x_{6}\right\}$. Since $e\left(S_{1}\right)=3$ and $x_{2} x_{6} \notin E(G)$, we may assume by symmetry that $S_{1}=\left\{x_{2}, x_{3}, x_{5}\right\}$. Then $e\left(S_{1}, S_{2}\right) \leq 8$, since $\left|N\left(x_{2}\right) \cap N\left(x_{5}\right) \cap V_{2}\right|=2$.

Now assume $e\left(S_{1}\right)=2$. Then $t\left(S_{1}\right) \leq 1$; otherwise by (2) and (3), $\left\{x_{1}, x_{7}\right\} \subseteq S_{1}$ and $x_{4} \notin S_{1}$, and we would have $e\left(S_{1}\right) \leq 1$. So it suffices to show that $e\left(S_{1}, S_{2}\right) \leq 7$, in which case $t\left(S_{1}\right)-2 e\left(S_{1}\right)+e\left(S_{1}, S_{2}\right) \leq 1-4+7<e\left(S_{2}, V_{1} \backslash S_{1}\right)$ (by (5)). If $t\left(S_{1}\right)=-1$ then by (4) and since $e\left(S_{1}\right)=2$, we have $S_{1}=\left\{x_{2}, x_{4}, x_{6}\right\}$, and so $e\left(S_{1}, S_{2}\right) \leq 7$ (since $\left.\left|N\left(x_{2}\right) \cap N\left(x_{6}\right) \cap V_{2}\right|=1\right)$. Suppose $t\left(S_{1}\right)=1$. Then by (1), $\left\{x_{1}, x_{7}\right\} \cap S_{1} \neq \emptyset$. So by symmetry assume $x_{1} \in S_{1}$. If $x_{4} \in S_{1}$, again by (1), $S_{1}=\left\{x_{1}, x_{4}, x_{7}\right\}$, and so $e\left(S_{1}, S_{2}\right) \leq 7$ (since $\left.\left|N\left(x_{1}\right) \cap N\left(x_{7}\right) \cap V_{2}\right|=0\right)$. So assume $x_{4} \notin S_{1}$. Then $x_{7} \notin S_{1}$, and so, $S_{1}=\left\{x_{1}, x_{2}, x_{5}\right\}$ or $S_{1}=\left\{x_{1}, x_{3}, x_{5}\right\}$ or $S_{1}=\left\{x_{1}, x_{3}, x_{6}\right\}$. In these cases we have $e\left(S_{1}, S_{2}\right) \leq 7$ (since $\left.\left|N\left(x_{1}\right) \cap N\left(x_{5}\right) \cap V_{2}\right|=\left|N\left(x_{1}\right) \cap N\left(x_{6}\right) \cap V_{2}\right|=0\right)$. Now suppose $t\left(S_{1}\right)=0$. If $x_{4} \in S_{1}$, then by (1) and since $e\left(S_{1}\right)=2$, exactly one of $\left\{x_{1}, x_{7}\right\}$, say $x_{1}$ (by symmetry), is in $S_{1}$; thus $S_{1}=\left\{x_{1}, x_{4}, x_{5}\right\}$ or $S_{1}=\left\{x_{1}, x_{4}, x_{6}\right\}$, and hence $e\left(S_{1}, S_{2}\right) \leq 7$ (since $\left.\left|N\left(x_{1}\right) \cap N\left(x_{5}\right) \cap V_{2}\right|=\left|N\left(x_{1}\right) \cap N\left(x_{6}\right) \cap V_{2}\right|=0\right)$. So $x_{4} \notin S_{1}$. Then since $t\left(S_{1}\right)=0$ and by (1), $\left\{x_{1}, x_{7}\right\} \cap S_{1}=\emptyset$. Hence, since $e\left(S_{1}\right)=2, S_{1}=\left\{x_{2}, x_{3}, x_{6}\right\}$ or $S_{1}=\left\{x_{6}, x_{5}, x_{2}\right\}$, and we have $e\left(S_{1}, S_{2}\right) \leq 7$ again (since $\left|N\left(x_{2}\right) \cap N\left(x_{6}\right) \cap V_{2}\right|=1$ ).

Finally assume $e\left(S_{1}\right)=1$. Then $x_{4} \notin S_{1}$, and so $t\left(S_{1}\right) \neq-1$ (by (4)). Moreover, $t\left(S_{1}\right) \neq 0$ as otherwise $S_{1} \subseteq\left\{x_{2}, x_{3}, x_{5}, x_{6}\right\}$ which implies $e\left(S_{1}\right) \geq 2$. So $1 \leq t\left(S_{1}\right) \leq 2$. If $t\left(S_{1}\right)=2$ then by (1), $S_{1}=\left\{x_{1}, x_{7}, x_{k}\right\}$, with $k \in\{2,3,5,6\}$; in these cases we can check that $e\left(S_{1}, S_{2}\right) \leq 5$, and so $t\left(S_{1}\right)-2 e\left(S_{1}\right)+e\left(S_{1}, S_{2}\right) \leq 2-2+5<e\left(S_{2}, V_{1} \backslash S_{1}\right)$ (by (5)). If $t\left(S_{1}\right)=1$ then by (1), exactly one of $\left\{x_{1}, x_{7}\right\}$, say $x_{1}$ (by symmetry), belongs to $S_{1}$. Since $e\left(S_{1}\right)=1, S_{1}=$ $\left\{x_{1}, x_{2}, x_{6}\right\}$, and so $e\left(S_{1}, S_{2}\right) \leq 6$. Hence $t\left(S_{1}\right)-2 e\left(S_{1}\right)+e\left(S_{1}, S_{2}\right) \leq 1-2+6<e\left(S_{2}, V_{1} \backslash S_{1}\right)$ 
(by $(5)$ ).

Therefore, we have shown that $V_{1}, V_{2}$ is the unique balanced bipartition of $V(G)$ such that $e\left(V_{1}, V_{2}\right)$ is maximal among all such partitions. So the constant $c$ in the following question satisfies $7 / 5 \leq c<13 / 4$.

Problem 4.1 What is the largest constant $c$ such that for any graph $G$ with $\Delta(G) \leq c \delta(G)$, if $V_{1}, V_{2}$ is a balanced bipartition of $V(G)$ with $e\left(V_{1}, V_{2}\right)$ maximum then $\max \left\{e\left(V_{1}\right), e\left(V_{2}\right)\right\} \leq$ $e(G) / 3$ ?

We conclude this paper with the following question of Bollobás and Scott.

Problem 4.2 (Bollobás and Scott [3]) What is the smallest constant $c(d)$ such that every graph $G$ with $\delta(G) \geq d$ has a balanced bipartition $V_{1}, V_{2}$ such that $\max \left\{e\left(V_{1}\right), e\left(V_{2}\right)\right\} \leq c(d) e(G)$ ?

Acknowledgment. We thank an anonymous referee for pointing us to reference [9].

\section{References}

[1] B. Bollobás and A. D. Scott, Judicious partitions of graph, Period. Math. Hungar 26 (1993) 125-137.

[2] B. Bollobás and A. D. Scott, Exact bounds for judicious partitions of graphs, Combinatorica 19 (1999) 473-486.

[3] B. Bollobás and A. D. Scott, Problems and results on judicious partitions, Random Struct. Alg. 21 (2002) 414-430.

[4] B. Bollobás and A. D. Scott, Better bounds for Max Cut, in Contemporary Comb, Bolyai Soc Math Stud 10, János Bolyai Math Soc, Budapest, 2002, pp. 185-246.

[5] B. Bollobás and A. D. Scott, Judicious partitions of bounded-degree graphs, J Graph Theory 46 (2004) 131-143.

[6] C. S. Edwards, Some extremal properties of bipartite graphs, Canadian J. math. 25 (1973) $475-485$.

[7] C. S. Edwards, An improved lower bound for the number of edges in a largest bipartite subgraph, in Proc. 2nd Czechoslovak Symposium on Graph Theory, Prague (1975) 167181.

[8] T. D. Porter, On a bottleneck bipartition conjecture of Erdös, Combinatorica 12 (1992) $317-321$.

[9] T. D. Porter and L. A. Székely, On a matrix discrepancy problem, Congressus Numerantium 73 (1990) 239-248.

[10] F. Shahrokhi and L. A. Székely, The complexity of the bottleneck graph bipartition problem, J. Combin. Math. Combin. Comp. 15 (1994) 221-226.

[11] B. Xu and X. Yu, Judicious k-partitions of graphs, manuscript submitted.

[12] J. Yan and B. Xu, Balanced judicious partitions of $(k, k-1)$-biregular graphs, manuscript submitted. 